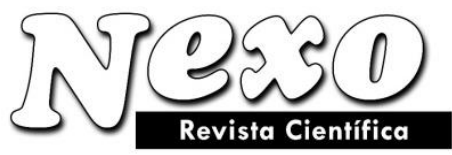

\title{
Análisis de restauración de la iglesia Santa Lucia de San Sebastián, Retalhuleu y revitalización del entorno inmediato
}

\section{Restoration analysis of the Santa Lucia church of San Sebastián, Retalhuleu and revitalization of the immediate environment}

\author{
Luis E. Mazariegos Meneses \\ Universidad de San Carlos de Guatemala, Programa de Maestría de Restauración de Monumentos \\ Históricos con especialidad en Centros Urbanos, Guatemala, Guatemala. \\ luismaza01@gmail.com
}

(recibido/received: 30-agosto-2021; aceptado/accepted: 23-noviembre-2021)

\begin{abstract}
RESUMEN
La Iglesia de Santa Lucía de San Sebastián Retalhuleu, Guatemala se encuentra abandonada, recibiendo las inclemencias del tiempo, con serios daños provocados por distintos factores, entre los cuales se encuentran la falta de mantenimiento a lo largo del tiempo, además de acontecimientos telúricos que han incrementado el deterioro, siendo el de 17 junio del año 2017 el último que dañó seriamente los vestigios de la fachada principal, haciendo colapsar el muro de esta, los revoques que aun existían, y exponiendo la mampostería original. Otro problema latente, lo constituyen las distintas intervenciones que se han hecho, las cuales han sustituido materiales originales, por materiales contemporáneos, sin ningún criterio apropiado. El funcionamiento espacial para el cual fue diseñado el edificio ha dejado de satisfacer sus necesidades primigenias. Su historia general plantea que han sido distintos los usos que se le han dado a lo largo del tiempo, siendo a principios del siglo XIX, primero ermita, para proteger la imagen de Santa Lucía, mandada a construir por el Señor Cristóbal Cochajil; para ser luego una iglesia. Posteriormente a ello otros variados usos, desde bodega para materiales, sala de reuniones para los feligreses y distintas organizaciones católicas, y actualmente abandonado en su totalidad, lo cual ha provocado que se le hagan las modificaciones necesarias para que estos puedan funcionar, todo ello carente de criterios que protejan la originalidad del edificio, y poniendo en peligro el valor patrimonial del mismo.
\end{abstract}

Palabras claves: Restauración; Patrimonio; Revitalización.

\section{ABSTRACT}

The Church of Santa Lucía de San Sebastián Retalhuleu, Guatemala is abandoned, receiving the inclement weather, with serious damage caused by different factors, among which are the lack of maintenance over time, in addition to telluric events that have increased the deterioration, being the one of June 17, 2017 the last one that seriously damaged the vestiges of the main façade, causing the wall of this to collapse, the plasters that still existed, and exposing the original masonry. Another latent problem is the different interventions that have been made, which have replaced original materials, by contemporary materials, without any appropriate standards. The spatial operation for which the building was designed has ceased 
to meet its original needs. Its general history states that the uses that have been given over time have been different, being in the early nineteenth century, first hermitage, to protect the image of Santa Lucia, ordered to be built by Lord Cristóbal Cochajil; to be later a church. In addition, there are other varied uses, from a cellar for materials, a meeting room for parishioners and various Catholic organizations, and currently abandoned in its entirety, which has caused the necessary modifications to be made so that this can work, all lacking criteria that protect the originality of the building, and endangering the patrimonial value of it.

Keywords: Restoration; Heritage; Revitalization.

\section{INTRODUCCIÓN}

Las edificaciones eclesiásticas consideradas patrimoniales son de gran valor histórico cultural para las presentes y futuras generaciones. Lamentablemente en muchos casos los deterioros debido a diferentes causas provocan la pérdida irreparable del valor patrimonial de éstas, siendo así que el presente trabajo de tesis de la propuesta de Restauración de la Iglesia Santa Lucía de San Sebastián, Retalhuleu y revitalización del entorno inmediato, tiene como objetivo general Diseñar un proyecto de restauración y revitalización urbana del entorno inmediato, de la Iglesia Santa Lucía del municipio de San Sebastián, del departamento de Retalhuleu, para contribuir a la conservación del patrimonio como un espacio de uso histórico-urbanocultural. Por lo cual el presente artículo pretende explicar la propuesta de restauración del bien inmueble, en el el cual plantea el proceso de investigación que permitió determinar la revitalización del entorno y la restauración del inmueble afectado por acontecimientos naturales, logrando determinar que actualmente se encuentra en un marcado abandono por la falta de mantenimiento, abandono, intemperismo y negligencia.

\section{ESTRUCTURA DEL MANUSCRITO}

\subsection{Aspectos generales sobre conservación de monumentos}

La restauración arquitectónica de edificios arqueológicos, se define como "La rama del conocimiento llamada arquitectura, entendiendo esta actividad en un amplio sentido, no sólo en lo concerniente a la definición tridimensional por medio de elementos que adquiere un valor representativo de esa época y eventualmente de varias en el caso que el edificio hubiera sufrido más de una reconstrucción, como se percibe la obligatoriedad actualmente de preservar esos ejemplos cada vez más escasos de arquitectura y de esa manera valor no sólo el espacio logrado, sino el manejo de los materiales para hacerlos realidad; incluyendo también su valor estético, es decir la particularidad que le reviste como un objeto artístico, un objeto que entraría a formar parte de la cultura, aunque su posición respecto de la cultura de los diversos grupos sociales en una sociedad variará debido a diversos factores, entre ellos la mayor o menor persistencia de ese objeto en la memoria colectiva, la o las formas en que dicho objeto es valorado y aceptado o no, el manejo que del mismo exista a nivel ideológico, político para mencionar apenas unas pocas variables". (Molina, 1975)

\subsection{Proceso metodológico para la investigación}

Serie de gráficas que forman parte del proceso metodológico para la toma de decisiones del proceso de investigación: (a) inicio de la metodología utilizada con el análisis histórico y físico (b) continuación con el estudio del estado actual (c) Las transformaciones llevadas a cabo durante la historicidad del inmueble. (d) toma de datos hasta las especificaciones técnicas.

Fuente: Elaboración propia 


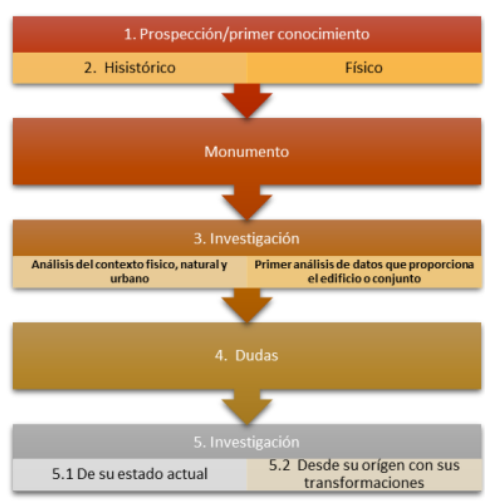

(a)

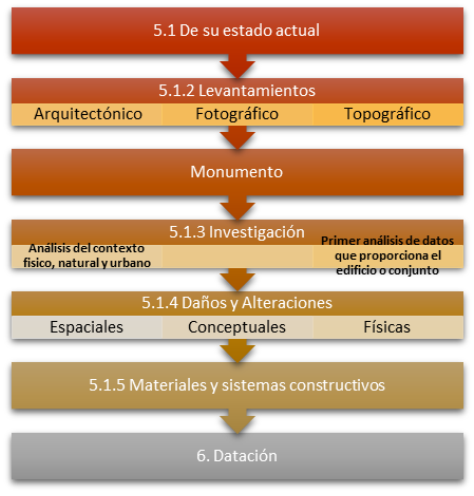

(b)

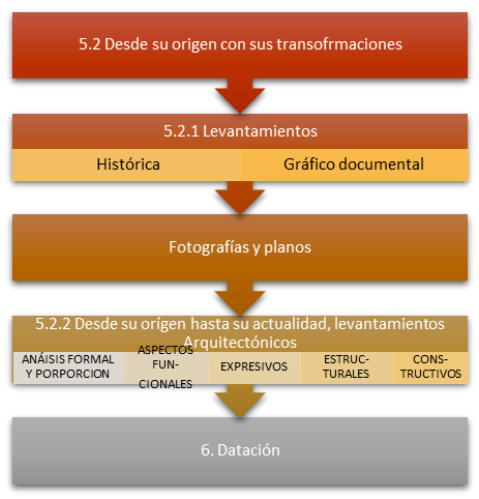

(c)

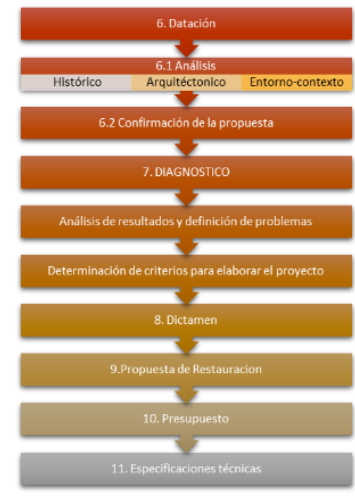

(d)

Figura 1 Proceso metodológico de investigación

Fuente: Elaboración propia

La metodología propuesta para la investigación del inmueble arriba descrita, inicia con un primer acercamiento, (1) en donde surgen las primeras incógnitas históricas y físicas del monumento, (2). Con esto se inicia el proceso de investigación (3), que incluye el contexto natural, urbano, y los datos que físicamente pueda proporcionar el edificio.

De este primer acercamiento, surgen incógnitas o dudas (4), que dan proceso a la investigación (5) con 2 líneas a seguir: la primera el estado actual (5.1) del monumento, y el segundo todos aquellos aspectos que han sido modificados desde su origen hasta la fecha actual (5.2).

En la etapa del análisis del estado actual (5.1), se realizarán los levantamientos arquitectónicos, fotográficos y topográficos (5.1.2), que nos permitirán establecer la volumetría y forma del edificio actual del monumento. Con estos datos, se procede a investigar y analizar (5.1.3) el entorno urbano, y los datos que proporcionaron los levantamientos, y que dictan la realidad del monumento espacialmente. Esto conlleva a poder determinar los daños y alteraciones (5.1.4) conceptuales, espaciales y físicas del inmueble, para poder llegar al análisis de los materiales y sistemas constructivos (5.1.5), que en conjunto permiten pasar a la datación (6).

Paralelo a ello, el proceso también establece el análisis de los aspectos que fueron modificados del edificio desde su origen hasta la época actual ( 5.2), este proceso también establece levantamientos desde históricos, gráficos documentales, (5.2.1) como imágenes, información de periódicos, entrevistas, planos, que permiten tomar una idea del edificio, desde sus etapas iniciales, y las modificaciones hechas, si es que existen, hasta su estado actual (5.2.2), que en conjunto también permiten pasar a la datación (6)

Los pasos arriba descritos nos permiten pasar al proceso de datación (6), el cual utilizando toda la información obtenida, se realiza el proceso de análisis (6.1), que permite establecer y confirmar la propuesta adecuada (6.2), esto junto con el diagnóstico del edificio (7), permite hacer el análisis de los resultados para definir el problema y los criterios a utilizar, y establecer el dictamen (8) y la propuesta de restauración (9), ya con esta información se procede a establecer el presupuesto general, que determina un costo en términos monetarios(10) y las especificaciones técnicas (11) que establecen la forma y métodos de desarrollo constructivo y proceso de restauración.

\subsection{Resumen histórico}


La historia acontece, en el área de la boca costa de Guatemala, dentro de lo que hoy se conoce como el departamento de Retalhuleu, algunos datos importantes que se encuentra es que uno de los primeros poblados en donde se llevaron las primeras batallas, fue el pueblo de Xetulul, que más tarde se le conoció como Zapotitlán, que en lengua Nahual, significa "lugar donde abundan los zapotes". (Van Oss, 1984)

En el año de 1537, lo que forma hoy la franja que limita a los ríos de Samalá y Tilapa fue entregada en calidad de título de tierra al señor Francisco Tajzoy, por la justicia de Santa Cruz del Quiché. (Robert Carmeck)

Ya para el año de 1554, se tuvieron los primeros intentos de evangelización, por parte de los Franciscanos. En 1565, son retirados por Fray Bernardino de Villalpando, a la llegada de la orden de los Dominicos, quienes son los que realizan los primeros trazos, de Calles y Avenidas, en lo que hoy es Retalhuleu. (Archivo General de Centroamérica, 1574)

La fundación de la Provincia de Zapotitlán, como parte de la población española, data de mediados del siglo XVI, entre los años 1560 a 1565, cuando se nombró a un alcalde mayor para la citada provincia. Según, Domingo Juarros, en el libro de Bocetos Históricos, de Ramón Serra, indica que antes del año de 1574 la jurisdicción de Guatemala llegaba hasta la provincia de Zapotitlán. (Archivo General de Centroamérica, 1574)

Para el año de 1581, el señor Juan de Estrada, quien fungía como alcalde Mayor de la región de Zapotitlán y Suchitepéquez, describe la forma de vida de los pueblos de indios, de las ceremonias, ritos, sacrificios, supersticiones, forma de vestir y los cambios que fueron sufriendo con la llegada de los españoles. Describió la parte de lo que hoy se le denomina "costa" como una tierra cálida con mucha humedad, llena de ríos, arroyos, lagunas pantanos, ciénagas, en donde la lluvia es abundante, con truenos y relámpagos, región de muchos movimientos telúricos. Describe así mismo, que el lugar, era montuoso, de muchos árboles, que impedían una vista clara de los pueblos cercanos. La abundancia de árboles, y otras especies de diferentes plantas, como el caco, maíz, frijoles, el camote, algodón entre otras, forma parte de la descripción.

Según el libro de bocetos históricos de Ramón Serra, describe que, en el año de 1635, el quien entonces fungía como presidente, el General Osorio, manda a los alcaldes mayores, a sacar a los españoles y ladinos de las poblaciones indígenas de la provincia de Zapotitlán, debido a desmanes que cometía contra estos. Esto provocó un gran resentimiento, sobre todo de los pobladores de la villa de San Sebastián Retalhuleu, quienes provocaron batallas campales, en contra de los ladinos de Retalhuleu. (Acuña, 1982)

Entre los años 1776 y 1781, el poblado de San Sebastián fue separado de la parroquia de Ostuncalco y agregado como a nexo a la parroquia de San Antonio Retalhuleu. (Robert Carmeck)

En el año de 1806, el pueblo de San Sebastián, según información del Archivo general de las indias, contaba con aproximadamente 596 tributarios, por lo que se solicita que se les otorguen las tierras del pueblo, ya extinguido, de San Luis Zapotitlán, "para poder repararse de los quebrantos sufridos durante el incendio del 3 de marzo de ese año. (Archivo General de Centroamérica, 1574)

En 1841, el entonces corregidor de Suchitepéquez, y del ingeniero Van Duchette, en 1852, indicaban que existía un camino que unía a Retalhuleu y Quetzaltenango (camino de San Luis), el cual pasaba por el puente y pueblo de San Sebastián. (Serra, 1970)

En el año de 1877, se concedió al municipio de Salcajá, Quetzaltenango, algunos terrenos que forman la mayor parte de ejido de San Sebastián. (Van Oss, 1984)

En el año de 1879, el entonces presidente de Guatemala el General Justo Rufino Barrios emite el Decreto, donde San Sebastián, pasa a ser municipio de Retalhuleu, el 11 de noviembre del mismo año. El 26 de 
enero de 1880, el ingeniero José Escobar, a petición de los pobladores, realiza las mediciones correspondientes para saber el área total del mismo. Debido a problemas de los mojones, al final, el área quedó, según sus mediciones de 44 caballerías, 53 manzanas, 7,403 varas cuadradas.

Para entonces se tiene conocimiento, que se crea el inmueble de la Iglesia de Santa Lucia, inicialmente Ermita, que fue construida por iniciativa del señor Cristóbal Cochajil, quien siendo de familia adinerada, y sin contar con familia, entre sus posesiones más preciadas, tenía una imagen de la Virgen de Santa Lucía. Al contar con los recursos monetarios, decidió iniciar el proceso de la construcción para salvaguardar la imagen, este lugar es considerado sagrado "chuc'aja", utilizado por sacerdotes mayas, para agradecer a las divinidades. (Palaj, 2019).

Tabla 1 Resumen cronológico de los acontecimientos más importantes suscitados des de 1784 hasta el año 2017, a la iglesia de Santa Lucia, de San Sebastián Retalhuleu, Guatemala.

\begin{tabular}{|c|c|}
\hline$\overline{\mathrm{AÑO}}$ & SUCESO \\
\hline 1784 & $\begin{array}{l}\text { Se tiene el primer dato, de la existencia de la Iglesia } \\
\text { de Santa Lucía, de san Sebastián, Retalhuleu. }\end{array}$ \\
\hline 1902 & $\begin{array}{l}\text { Daños en la cubierta, por la erupción del volcán } \\
\text { Santa María. }\end{array}$ \\
\hline 1944 & $\begin{array}{l}\text { Nuevamente, se sufren daños en la cubierta, por } \\
\text { sismo, que término de destruir la cubierta. }\end{array}$ \\
\hline 1947 & Creación del primer comité, pro-restauración \\
\hline 1972 & Destrucción total de la cubierta, y abandono total \\
\hline 1980 & Pavimentación del atrio, actualmente $3^{\mathrm{a}}$. Calle. \\
\hline 1983 & $\begin{array}{l}\text { Creación de un nuevo Comité de Restauración. } \\
\text { Acercamiento con el Instituto de Antropología e } \\
\text { Historia. Intento de primera propuesta de } \\
\text { restauración. }\end{array}$ \\
\hline 2006 & $\begin{array}{l}\text { Intervenciones y cambios en piso y cubierta, sin } \\
\text { ningún acompañamiento profesional. Creación de un } \\
\text { tercer, comité pro-restauración. }\end{array}$ \\
\hline 2017 & $\begin{array}{l}\text { Sismo, que termina de destruir los revoques de la } \\
\text { fachada principal, y proceden a la delimitación del } \\
\text { espacio de la iglesia, para evitar cualquier acceso por } \\
\text { el estado de deterioro. }\end{array}$ \\
\hline
\end{tabular}

Fuente: (Elaboración propia, 2020).

\section{ANÁLISIS DEL ENTORNO Y CONTEXTO}

\subsection{Delimitación del entorno inmediato}

El inmueble, se encuentra ubicado sobre la $5^{a}$. Avenida y la $3^{\text {a }}$ Calle de la Zona 3 o Cantón Paoj del municipio de San Sebastián Retalhuleu, la propiedad se encuentra registrada bajo Finca Urbana Número 8189, Folio 113, Libro 44 de Retalhuleu, que pertenece a la Municipalidad de Retalhuleu de San Sebastián. Las dimensiones correspondientes, son: norte mide 3250 metros, colinda con el terreno del señor Sebastián Poz, y con el terreno municipal donde se encuentra un tanque público del Cantón Paoj; al oriente 13,820 metros, colinda con la $5^{\mathrm{a}}$. Avenida de la Zona 3; al poniente mide 17,995 metros, colinda con los terrenos de los señores Andrea Ovando, Ignacio Reynoso, Vicente Chepemo, José Cuxevà, Domingo Cuyuj, Isidro Xicara y Casiano Canux, y al sur mide 2840 metros, colinda con la $3^{\text {a }}$ Calle de la Zona 3. 


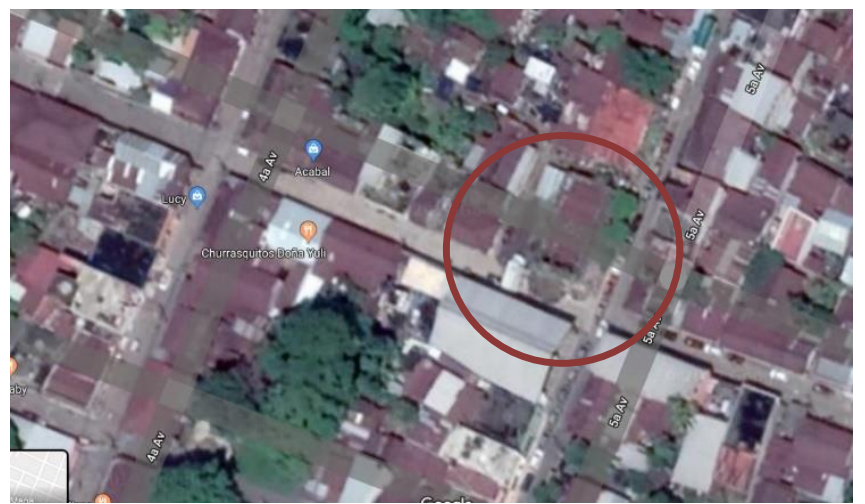

Figura 1 Vista área de la ubicación de la Iglesia de Santa Lucia, dentro del círculo.

Fuente: (GOOGLE MAPS. 2019)

\subsection{Características del entorno inmediato}

El entorno inmediato, se encuentra predominado por construcciones con características de uso actual, sin orden en la imagen urbana, y de uso mixto tanto comercial como vivienda. En lugares puntuales, que se podrán verificar en el plano de conjunto, se puede identificar casas de un nivel, de características vernáculas que poseen cimientos de piedra morlón, sobreseimiento de piedra o adobe, muros de mampostería de madera, cubierta de lámina de zinc o teja de barro, con estructura de madera. Las edificaciones existentes durante la investigación se puedo determinar que son de uso mixto, las cuales aún mantienen ciertas características originales, no pudiéndose determinar con certeza los cambios realizados a lo largo del tiempo.

Sobre la $3^{\mathrm{a}}$. Calle, la edificación que más predomina en el entorno urbano es la estructura de metal y la cubierta lámina acanalada, de la cancha polideportiva municipal, que se encuentra ubicada hacia al sur, frente a la Iglesia, que actualmente funciona como área recreativa, con características de uso actual en su totalidad. Como se podrá observar en el análisis fotográfico, de la $3^{\text {a }}$. Calle.

Sobra la $5^{\mathrm{a}}$. Avenida, en la morfología de las edificaciones son variadas, siendo estas en su mayoría, con características de uso actual, de 1 a 2 niveles, de uso mixto (comercio/vivienda), de características vernáculas de un nivel. Como se podrá observar en el análisis fotográfico, de la 5 a . Avenida.

\subsection{Análisis fotográfico del estado actual del edificio y su entorno}

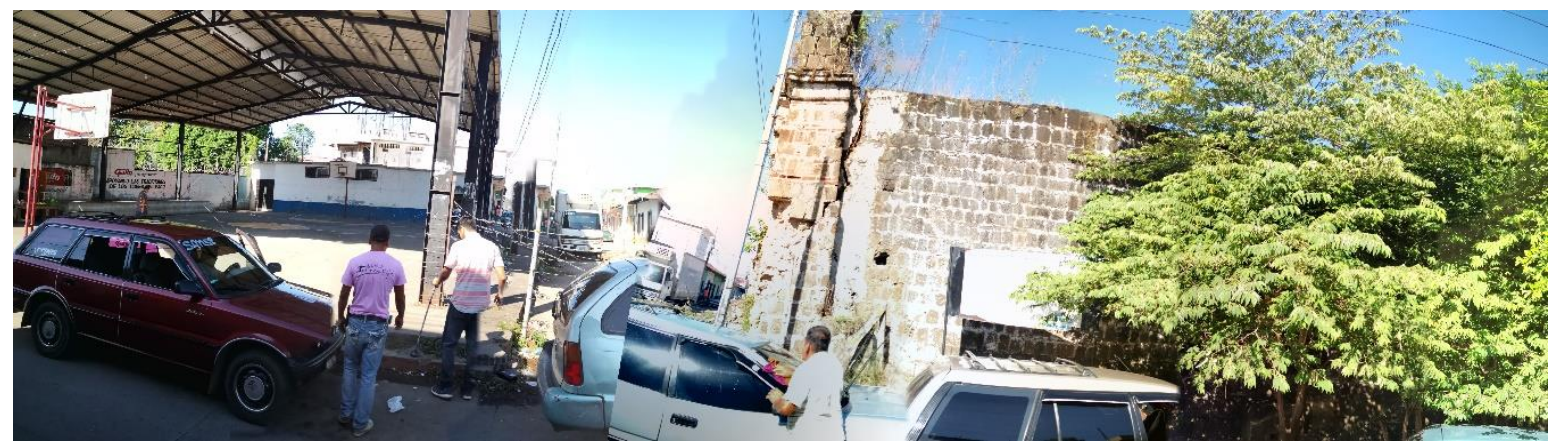

Figura 2 las intervenciones contemporáneas son evidentes, tal como se aprecia la estructura, que forma parte de la cubierta de la cancha polideportiva en el lado sur de la edificación, un elemento masivo, con características de uso actual, que, según la investigación, formaba parte del atrio de la iglesia, frente al monumento en estudio.

Fuente: (Elaboración propia,2021) 


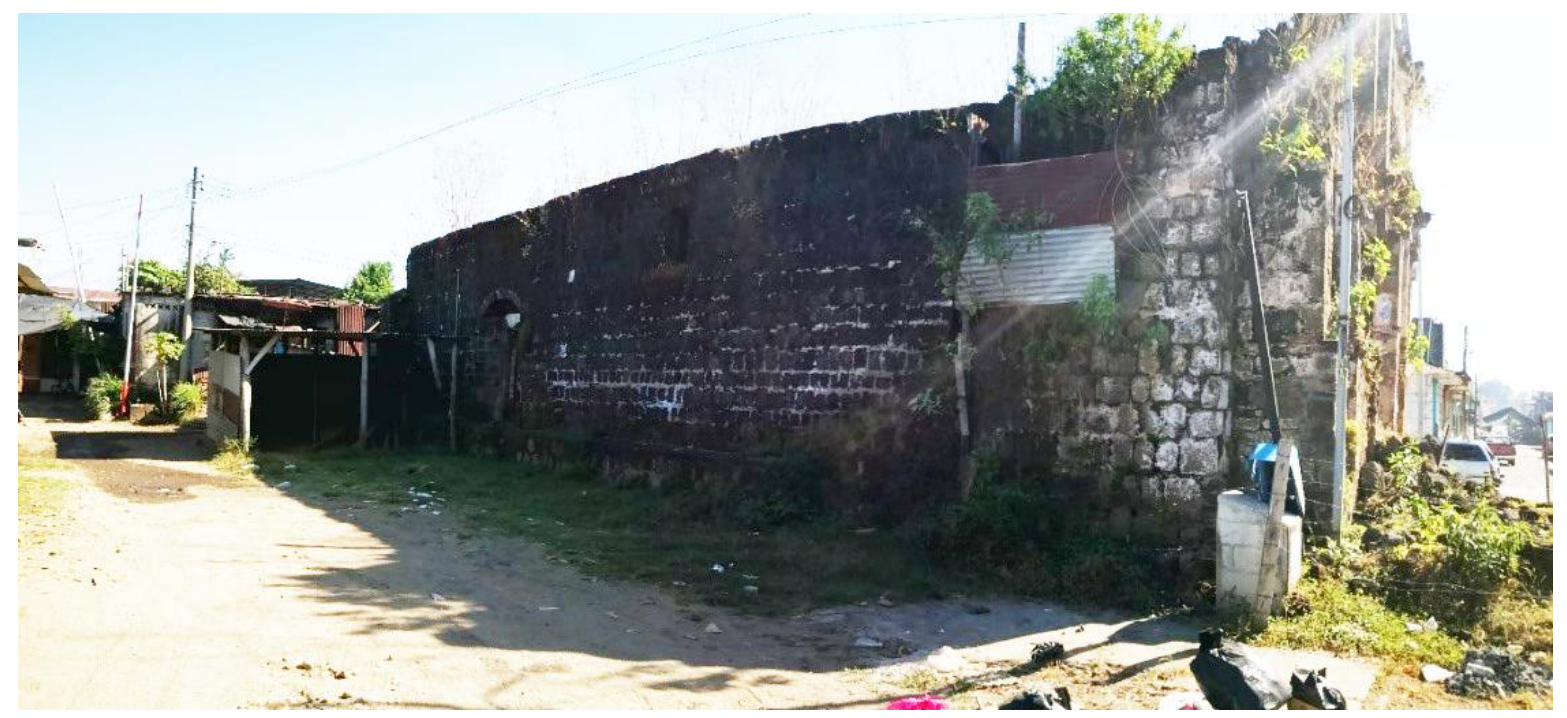

Figura 3 En la imagen se puede apreciar el lado oeste, de la Iglesia de Santa Lucía y el estado actual en cuanto al abandono y la macro flora es evidente.

Fuente:(( Elaboración propia,2021)

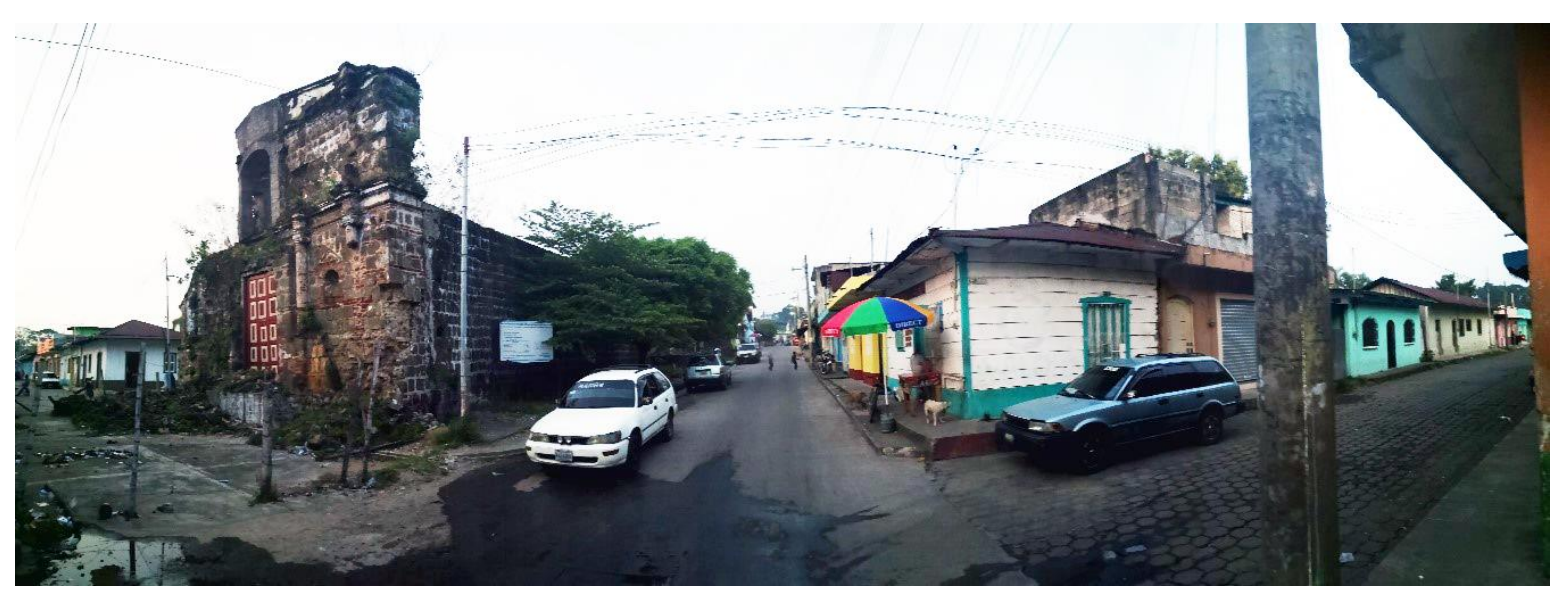

Figura 4 En la imagen se aprecia el estado actual de la fachada principal del lado sur de la Iglesia de Santa Lucia, además del contexto urbano inmediato, la tipología arquitectónica actual junto con la contaminación visual debido a mobiliario urbano, basura etc.

Fuente: (elaboración propia, 2021)

\section{RESUMEN DE LOS DAÑOS Y ALTERACIONES DEL EDIFICIO}

Para este artículo se tomó la decisión de hacer un resumen de los daños y alteraciones del edificio mediante la presentación de los mismos en fotografías que se encontraron durante el proceso de investigación y siguiendo la metodología propuesta, para lo cual se decide presentar la fachada principal, una fachada lateral además de 1 imagen del interior, lo cual permite tomar una visión general de cómo se encuentra actualmente el inmueble. 


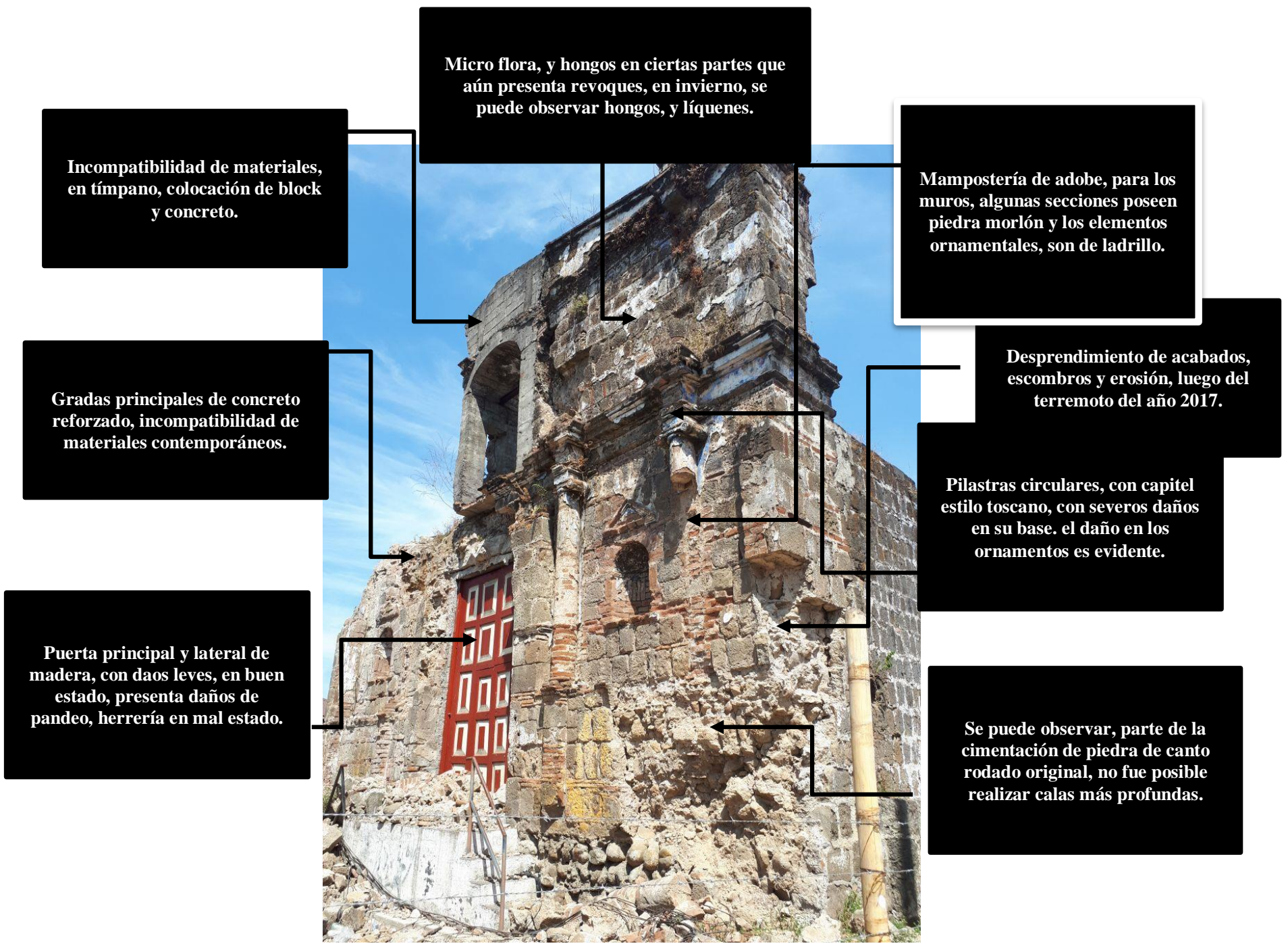

Figura 6 Fachada principal, iglesia Santa Lucia y el análisis de daños y alteraciones.

Fuente:( Elaboración propia, 2021) 
Herraría en mal estado,

herrumbre, faltante de piezas de madera en VENTANAS.

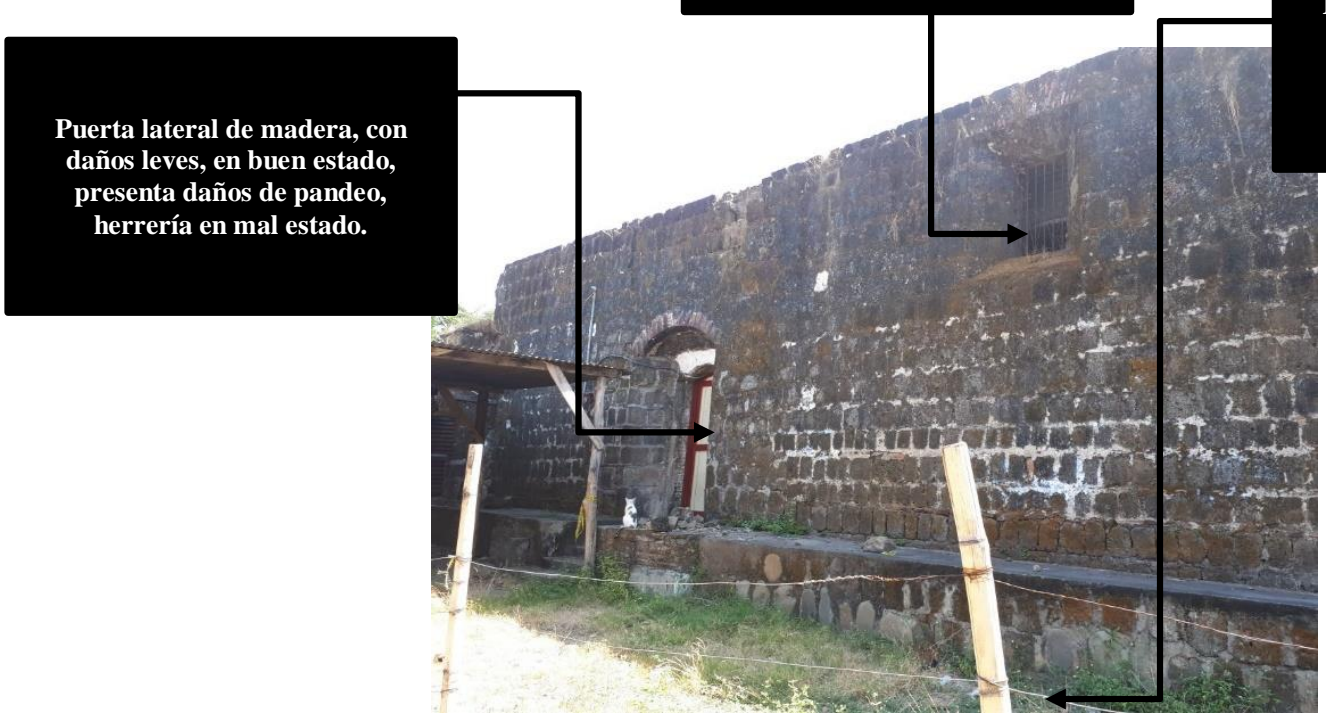

Micro flora, y macro flora, en invierno es más evidente, la conformación de hongos y líquenes.

Figura 7: Muro lateral Este, de iglesia santa lucia

Fuente: (Elaboración propia, 2021)

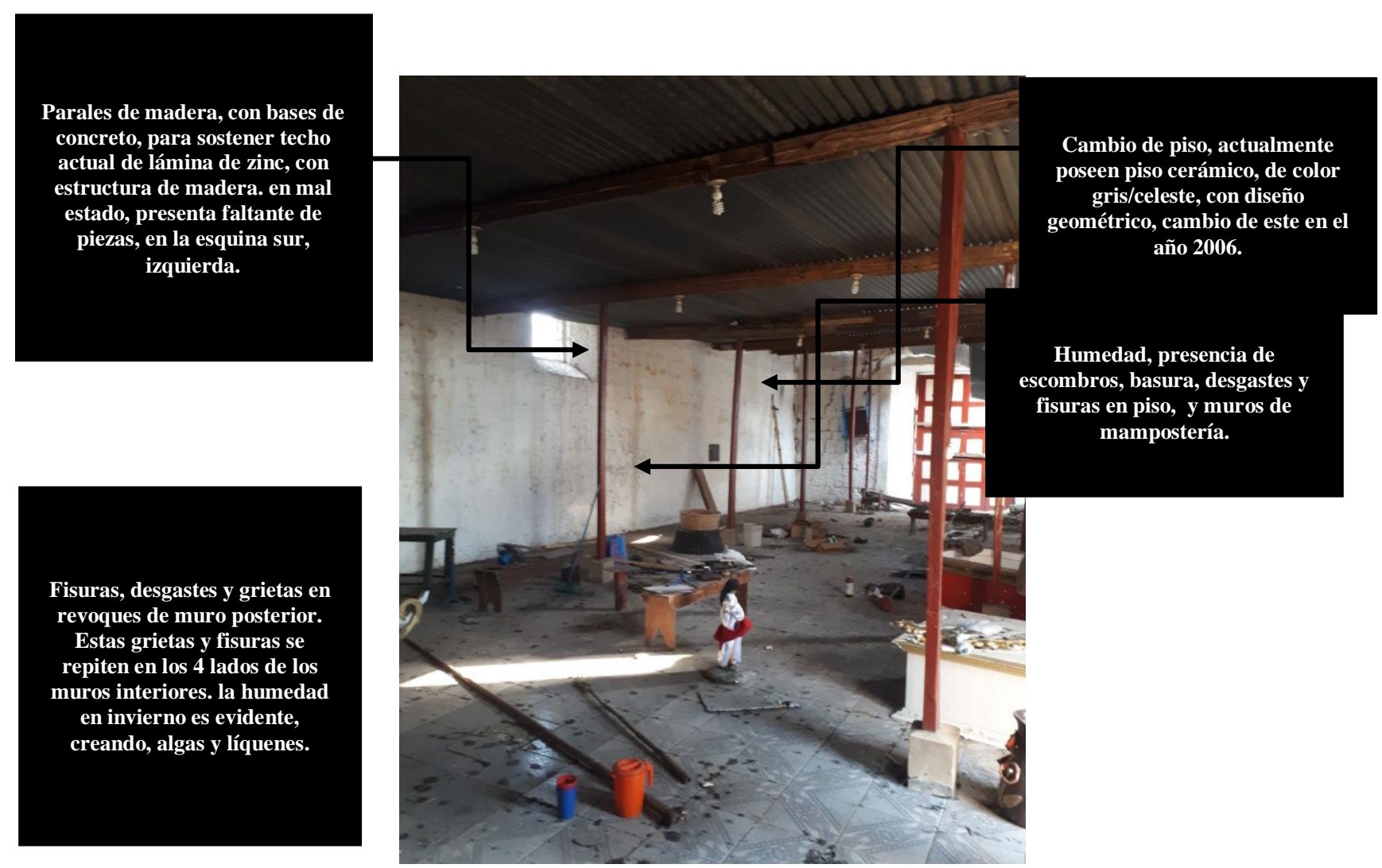

Figura 8: Área interior de la Iglesia Santa Lucia, vista desde el púlpito

Fuente: (Elaboración propia,2021) 


\section{DIAGNÓSTICO}

\subsection{Medios}

En el caso de la Iglesia de Santa Lucía de San Sebastián Retalhuleu, el medio es si es la edificación misma, partiendo que antiguamente fue el centro de todas las actividades religiosas que se desarrollaban en el municipio.

\subsection{Causas}

Siendo una edificación que, según las investigaciones, data del siglo XVII, con una edad aproximada de 300 años, es evidente que los materiales empleados han sufrido desgastes, fatigas, aunado al factor tiempo, es concluyente que el primer factor de las causas del daño a la edificación son los terremotos, siendo el del 14 de junio del año 2017, el último terremoto que destruyó el edificio, dejándolo en el estado actual. Aunado a ello el intemperismo, la falta de manteamiento preventivo, ausencia o mal seguimiento a un programa de mantenimiento, no reparar inmediatamente lesiones o fallos que consecuentemente han degenerado el edificio, abandono del inmueble, aumentan la continuidad del deterioro en el inmueble, provocando daños constantes , así como la vibración provocada por el paso de los vehículos que transitan sobre la $3^{\mathrm{a}}$ Calle de la Zona 1 de San Sebastián, Calle aledaña la Iglesia de Santa Lucía de San Sebastián Retalhuleu.

\subsection{Efectos}

Es evidente los daños, sobre todo en la fachada principal cuyos muros agrietados, desprendimiento de revoques, faltante de acabados, daños en los elementos ornamentales, el cambio de la cubierta actual, por una cubierta improvisada, deteriorada actualmente, muros laterales y posteriores, con fisuras y grietas, desprendimiento de acabados por fallas mecánicas, cambio del cien por ciento del piso original, por piso cerámico en el área interior de la única nave del edifico, integración de materiales que no son compatibles con los originales, como se evidencia en el concreto colocado en el tímpano de la fachada principal sur.

\subsection{Deterioros}

Sobre todo en la fachada principal se han localizado fisuras y grietas, desprendimiento del material compositivo, dejando vestigios de este, en el área el colapso parcial del tímpano de la iglesia, y más del $90 \%$ del material compositivo de algunos elementos ornamentales, debido al último sismo del año 2017, además de los daños arriba descritos; los muros exteriores y contra fuertes de la iglesia, también están expuestos a un constante deterioro por agentes biológicos (orina y heces fecales humanas), basura y vandalismo.

\section{EL PROYECTO DE RESTAURACIÓN}

Luego del análisis realizado, se establecieron las directrices que se deberán tomar, tomando en cuenta cada uno de los aspectos analizados, desde el entorno urbano, así como la recuperación del bien inmueble para proveer las directrices necesarias para su puesta en uso nuevamente, para lo cuál se describen las propuestas en cada uno de estos aspectos analizados: 


\subsection{Entorno urbano}

Durante el proceso de investigación, se pudo determinar que actualmente, se encuentra funcionando un grupo organizado de taxistas, que prestan servicio a la población. Este elemento social, se tomó en cuenta para el proceso de aceptación del proyecto, ya que fue evidente, que ellos ven el lugar como un predio que les permita realizar su empresa de manera más efectiva. Por lo que, para lograr un proyecto integral, se tomara en cuenta este servicio, adecuándolo al contexto urbano del proyecto, realizando y diseñando el mobiliario urbano y los espacios, que permitan un adecuado uso, y que pueda articularse con el monumento, dándole un aspecto estético y arquitectónico, adecuado, y funcional.

\subsection{Recuperación del atrio y cruz atrial}

Sergio Palaj, durante la entrevista habló sobre la existencia de la cruz atrial en el antiguo atrio de la iglesia, y las gradas de la iglesia, diseñadas de una manera que permitían acceder a este espacio. Por lo que en la propuesta se tomarán en cuenta estos 3 aspectos, además de hacer un cambio de textura, en todo el contexto y alrededor de la iglesia, proponiendo liberar en la parte posterior del lado norte, que lo conforman elementos que alteran el contexto urbano del edificio. Con esto se pretende crear el mobiliario urbano adecuado, además de integrar la vegetación, como parte del diseño paisajístico de la propuesta. Esto se sustenta, con el hecho de que esta, permite espacios con una adecuada temperatura, además de la mejora del aspecto visual. Dentro de los elementos que se integraran en el este espacio, están luminarias, bancas techadas, jardineras, rampas, controladores de tráfico, área para parqueo de taxis, gradas de acceso y la cruz atrial, con estos elementos se recupera el espacio perdido y se diseña adecuadamente para mejorar la imagen urbana y darle importancia al edificio de la Iglesia de Santa Lucía.

En cuanto a las gradas de concreto reforzado y baranda metálica actual, que se encuentran en el acceso principal de la iglesia, estas se liberarán en su totalidad, y se propondrá un diseño que permita acceder al atrio, permitiendo una articulación más adecuada y la recuperación de ese espacio necesario para la iglesia.

\subsection{Campanario y Sacristía}

En sus inicios, según la investigación, existía una campana, que actualmente se encuentra en el patio exterior de la iglesia de San Sebastián Mártir, esta luego de uno de los sismos, se quebró, y quedó el vestigio, que se encuentra abandonada. Por lo que, como parte del proceso, se planteó diseñar un campanario, en la parte exterior junto a un área que conformará la sacristía, que permite no solo la colocación de una campana nueva en un segundo nivel, sino que en el primero se crea un área que permite la exposición de la campana original. El resto del inmueble permitirá acomodar las áreas para secretaría, oficina parroquial, los muebles de escritorio, archivos y un servicio sanitario, como parte de los elementos compositivos arquitectónicos y espaciales, además del diseño de una rampa de ingreso para el uso de personas con capacidades especiales, y que permita el ingreso hacia la iglesia desde la entrada lateral del muro este.

\subsection{Fachada principal y elementos ornamentales}


La fachada principal y sus elementos ornamentales, es la que más daños tiene, por lo que se planteó reintegrar los elementos ornamentales, dejando evidencia de su restauración, además de integrar nuevamente el tímpano de la fachada. La consolidación de los elementos en mal estado, daños, fisuras y grietas, además de recuperar los elementos compositivos originales, y los faltantes con materiales compatibles y sistemas constructivos amigables a los actuales. La recuperación de la fachada será en un aspecto volumétrico total, para recuperar el ícono arquitectónico del volumen, y la recuperación volumétrica de los elementos ornamentales más representativos, pilastras, capiteles estilo toscano, molduras, arquitrabes, entre otros elementos, basados en el análisis de sitio, fotografías antiguas y vestigios encontrados in situ.

\subsection{Muros laterales y posteriores}

El material compositivo de estos es piedra morlona, por lo que la propuesta consistió en dejar el material expuesto, aplicando un programa de mantenimiento y liberación de micro y macro flora, consolidación de fisuras y grietas, y un programa continuo de mantenimiento adecuado para su protección.

\subsection{Piso y muros interiores}

En cuanto al piso interior, se planteó el análisis profundo, con calas para determinar si aún se encuentran vestigios de los pisos originales. Así mismo se propondrá la liberación del piso actual de cerámica, por un piso con las mismas características del original.

En cuanto a los muros interiores, se planeó la liberación los revoques en mal estado, y se propone la integración de estos, con las mismas características del material compositivo de color blanco.

\subsection{Cubierta y estructura de techo}

Se formuló la liberación total de la estructura y cubierta actual, así como las bases de concreto y parales de madera. Se propuso una estructura de madera sin cielo falso, con cubierta de lámina de fibra cemento, con elementos de teja natural, para disminuir el peso del sistema tradicional de teja. Este elemento deberá integrarse en el muro posterior, con un dintel, y una viga encajuelada con ladrillo tuyuyo dentro de la cual se fundirá una viga solera de concreto reforzado, con pines, para anclar las tijeras sobre sobre los muros actuales. Ver Detalle 1, en Planos de Detalles. En este se integrarán las luminarias y sistemas eléctricos, adosados a los muros actuales con elementos transicionales de madera, para colocar la tubería. Para las bajadas de agua pluvial, se diseñaron gárgolas, que están conectadas a canales de metal en la parte interior.

\section{CONCLUSIONES}

- La propuesta de Restauración de la Iglesia de Santa Lucia de San Sebastián Retalhuleu y la revitalización del entorno inmediato ha sido favorecida, en base a los procesos que se requirieron para realizar dicho trabajo, lo cual ha consistido en la investigación, registros, trabajos de campo; localizando las áreas más vulnerables para realizar una pronta acción.

- Siendo la Restauración de la Iglesia de Santa Lucia el tema principal de investigación, con base en los requerimientos presentados para su aprobación en la Escuela de Postgrado de 
la Facultad de Arquitectura, Maestría en Restauración de Monumentos, se realizaron todos los pasos para cumplir una restauración científica, en donde se expone la importancia y permanencia del monumento, y las condiciones al que se expone.

- Cada actividad fue registrada, creando la base para sustentar la propuesta de restauración, consistente en: registros investigados, entrevistas, fechas de acontecimientos relevantes, levantamientos fotográficos, topográficos, análisis de sitio, condiciones actuales de la edificación, riesgos presentes, condiciones del estado actual, intervenciones recientes y pasadas.

- El conocimiento histórico del edificio permite establecer cronológicamente, a través de la investigación, todos aquellos acontecimientos históricos, alteraciones, cambios o modificaciones que se realizaron, así mismo poder entender aquellos fenómenos, naturales y culturales, que han modificado el estado de este hasta la actualidad.

- La Iglesia de Santa Lucía de San Sebastián Retalhuleu, luego del análisis realizado, se puede establecer que se encuentra en un estado de precariedad; este se crea luego del análisis realizado al inmueble mediante las fichas de diagnóstico: daños, alteraciones, además de constituir los materiales y sistemas constructivos; ya que se detectaron carencias en la consolidación del material compositivo de la fachada sur y la falta de una cubierta que proteja al inmueble del intemperismo, además de la falta de un adecuado manteamiento y el abandono en que se encuentra, lo que provoca que disminuyan las garantías de seguridad por debajo de un nivel considerado adecuado, pero sin llegar a poner en peligro la estabilidad del sistema constructivo o del edificio y por lo tanto su funcionalidad y habitabilidad.

- Dentro de estos elementos, además de la urgente consolidación de la fachada principal, se propuso la recuperación volumétrica del edificio para volver a darle una lectura íntegra al inmueble. Esto y la necesidad de poder recuperar el espacio del contexto inmediato, lleva a la necesidad de diseñar una cubierta que permita ponerla en funcionamiento nuevamente.

- En la actualidad es necesario contar con áreas alternas, como una sacristía, además de secretaría y servicios extras, que permiten que el edificio funcione adecuadamente, por lo que en respuesta a ello se diseña un área que cumple con los requisitos espaciales para las funciones administrativas propias de una iglesia, además que se incorpora un espacio para reestablecer la campana en una torre diseñada para dicho propósito y exponer la antigua como parte de los vestigios e historia de este.

- La Iglesia de Santa Lucía es un bien cultural, protegido por el Estado de Guatemala, cuyo valor arquitectónico, histórico y patrimonial, debe ser protegido, sumando los esfuerzos necesarios de todas las instituciones gubernamentales que este requiera para dicha labor.

- El estado actual del edificio, su contexto y entorno urbano, posee un daño de moderado a severo, presentando la fachada, los elementos ornamentales y la morfología del elemento arquitectónico, las áreas donde mayor daño ha tenido el edificio, siendo las causas; primero por la falta de manteamiento, segundo por la carencia de un presupuesto adecuado para dichos trabajos. 
- El intemperismo y los acontecimientos telúricos a lo largo de la historia del edificio han sido otros factores fundamentales para el deterioro y destrucción del edifico, llegando a colocar al mismo, en el estado actual, por lo que en consecuencia y de manera urgente debe realizarse una propuesta de recuperación, restauración y rehabilitación del entorno, para su salvaguarda.

- La recuperación del espacio urbano y contexto inmediato, por sí sola no funciona, cada espacio fue diseñado para articular al inmueble como elemento central y recuperar la funcionalidad de este y estos serán complemento del funcionamiento diario, para uso de la población y de las personas que se encuentren en el contexto inmediato.

\section{REFERENCIAS}

Acuña, R. (1982). Relaciones geográficas del siglo XVI. México: Universidad Autonoma de Méixico, Instituto de investigaciones antropológicas.

Archivo General de Centroamérica. (1574). Guatemala.

Baca, G. (2007). Fundamentos de Ingeniería Económica (4a. ed.). México D.F.: McGraw-Hill.

Mario Ceballos Espigares. (1995). Conservacion de Monumentos. GUATEMALA: Universidad de San Carlos de Guatemala.

Garza, J. (2014). Análisis multicriterio de puntos de inflexión de precio en el mercado de divisas. San Nicolás de los Garza, Nuevo León: Universidad Autónoma de Nuevo León.

Gomero, N. (2014). Análisis económico de los impuestos: Impacto en la rentabilidad de las inversiones. Quipukamayoc, 79-87.

Instituto Nicaragüense de Investigaciones y Estudios Tributarios. (2015). Balance de la Ley de Concertación Tributaria. Managua, Nicaragua: INIET.

La Gaceta. Diario Oficial. (2013). Decreto 01-2013. Reglamento de la Ley No. 822, Ley de Concertación Tributaria. Managua: La Gaceta.

Molina, A. (1975). La restauracion arquitectónica de edificios arqueológicos. Colección Científica. México: INAH.

Palaj, S. (Martes 4 de Diciembre de 2019). Entrevista sobre la Iglesia de Santa Lucia y su Historia. (A. L. Mazariegos, Entrevistador)

Robert Carmeck. (s.f.). Quichean Civiliation/The etnohistoric, etnographic and archeolical resources. California USA.: University of California Press. 
Rosillo, J. (2005). La inflación: ¿Elemento inocuo en las decisiones de inversión? Forum Empresarial, 20-46.

Ruiz, J. (1992). Capital y depreciación: Una aproximación endógena. Valencia, España: Universitat de Valencia.

Serra, R. (1970). Bocetos históricos de Retalhuleu. Retalhuleu.

Van Oss, A. (1984). Pueblos y parroquias de Suchitepéquez colonial. Guatemala: Revista Mesoamericana Año 5 Número 7.

\section{SEMBLANZA DE LOS AUTORES}

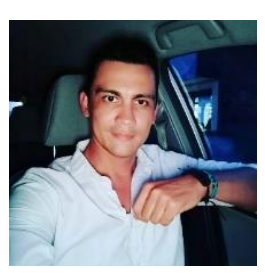

Luis E. Mazariegos-Meneses: Obtuvo el grado de Arquitecto en la de San Carlos de Guatemala, donde actualmente es profesor interino en el curso de Patrimonio Cultural, del centro universitario de Retalhuleu. Desarrolló sus estudios de maestría de Restauración de Monumentos en la misma universidad y obtuvo también el título de Magister Atrium en Evaluación y control ambiental, en la Universidad Mariano Gálvez de Guatemala. Ha sido consultor para diferentes instituciones de gobierno, se ha desarrollado como catedrático en de la Facultad de arquitectura de la universidad Mariano Gálvez, por 14 años. Actualmente es consultor para el Fondo de la Vivienda de Guatemala, socio fundador del Consorcio 3S Arquitectos y propietario de la empresa A+ de arquitectura por más de 16 años. 